\title{
SiC Devices for Renewable and High Performance Power Conversion Applications
}

\author{
Ibrahim Abuishmais and Tore M. Undeland \\ Department of Electric Power Engineering, Norwegian University of Science and Technology (NTNU), 7491 Trondheim, Norway \\ Correspondence should be addressed to Ibrahim Abuishmais, ibrahim.abuishmais@elkraft.ntnu.no
}

Received 27 December 2011; Accepted 6 February 2012

Academic Editor: Henry S. H. Chung

Copyright ( $) 2012$ I. Abuishmais and T. M. Undeland. This is an open access article distributed under the Creative Commons Attribution License, which permits unrestricted use, distribution, and reproduction in any medium, provided the original work is properly cited.

The unique properties of $\mathrm{SiC}$ devices enable substantial improvement of existing power conversion systems. SiC devices offer lower conduction and switching losses which increases converter efficiency. With high switching speed ability, employing SiC is expected to reduce weight and cost of conversion systems. This paper investigates the potential impact of SiC devices on renewable energy applications.

\section{Introduction}

Renewable energy system designers have been striving to achieve higher efficiency and lower cost systems. These gains will translate directly into higher adoption of renewable energy sources. SiC devices offer, compared to silicon, a higher voltage devices with lower on-state resistance and faster switching speed. This boosts converter efficiency by reducing conduction and switching losses. For example, photovoltaic inverters can achieve smaller size, lighter weight, and more cost-effective designs utilizing SiC. For example, inverter based on SiC JFET and Schottky diode has achieved efficiency up to $99 \%$ [1]. With such developments, solar energy price can be brought to comparative price level with conventional energy source. The high efficiency and cost effective SiC-based solution also benefits other applications including wind power and hybrid vehicle. Along with $\mathrm{SiC}$, $\mathrm{GaN}$ is another wide band-gap material that gained a lot of research interest in the last decade. GaN-based MOSFETs with extremely low on-resistance are already reported [2, 3]. These developments in wide band-gap transistors are expected to dominate the ever-growing renewable energy production systems in the near future.

\section{SiC Technology and State-of-the-Art}

2.1. Introduction. Due to the high critical electrical field for breakdown, SiC devices feature higher breakdown voltage. This high critical electric filed (ten times higher than silicon) allows for thinner devices and thus lower on-resistance. Moreover, $\mathrm{SiC}$ has the capability of high-temperature operation without sacrificing the switching speed or significantly increasing conduction loss, all of which make them very potential in the high-power, high-voltage, high-frequency, and high-density applications. This fits the requirements of renewable energy applications. However, methods of driving some of these devices, for example, JFET, are different from the conventional Si-MOSFET or BJT. Therefore, the acceptance of this type of devices depends on the understanding of its switching behaviour and the ability to design a proper driving circuit.

2.2. SiC Devices. Several SiC devices were demonstrated. Unipolar devices combine fast switching speed with low on-state loss. However, as device blocking voltage increases $(>3 \mathrm{kV})$, resistive voltage drop over the device drift region prohibitively increases. Hence, these devices are suitable 


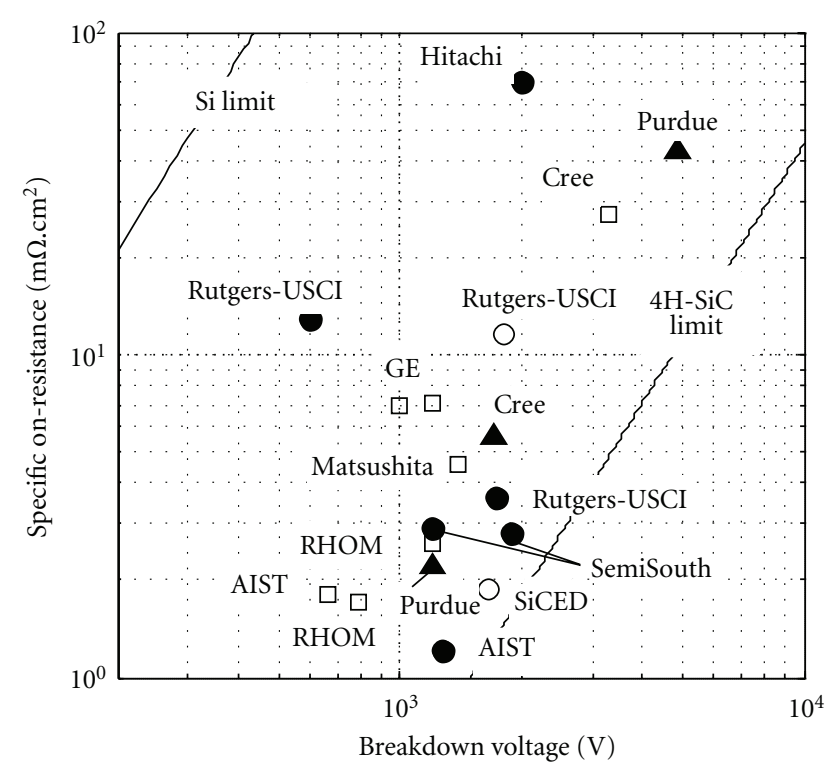

Figure 1: Specific on-state resistance of recent SiC power device, where $\square$ MOSFET, $\boldsymbol{\Delta}$ SBD, o normally-on JFET, and • normally-off JFET.

for medium voltage applications. Among unipolar devices Schottky didoes, JFETs, and MOSFETs are available. Schottky barrier diodes (SBDs) are made by utilizing the rectifying contact between a metal and device drift region. In case of silicon the high resistance of the drift region limits realizing high breakdown voltage devices and thus available devices are limited to $100 \mathrm{~V}$. In the case of $\mathrm{SiC}$, the higher breakdown field leads to lower drift region resistance. This enables higher breakdown voltages with lower losses making SiC-SBDs with breakdown voltage of $600-1700 \mathrm{~V}$ achievable. With the continuous development of the Schottky rectifiers, specific on-state resistance is already approaching the theoretical unipolar limit of SiC. Figure 1 shows some reported didoes with remarkably low on-resistance [4-6]. Nowadays, SBDs are commercially available from several manufactures, for example, Cree, Infineon, SemiSouth, STMicroelectronics, and Rohm.

JFET combines low on-state resistance with fast switching speed. Moreover, this device is free from reliability issues related to the gate oxide and temperature-dependent threshold voltages drifts. These reasons have made JFET one of the most successful devices within SiC technology [7]. The JFET is conventionally fabricated as a normally-on device. However, normally-off characteristic is achievable especially with the high built-in voltage $(\sim 3 \mathrm{~V})$ observed at $\mathrm{SiC}$ pn junction. Recent reported JFETs with $1.2 \mathrm{kV}$ breakdown voltage have specific on-resistance of only $2.47 \mathrm{~m} \Omega \mathrm{cm}^{2}$ and $2.9 \mathrm{~m} \Omega \mathrm{cm}^{2}$ for normally-on and normally-off devices, respectively [8]. On the other hand, MOSFETs are preferred by designers due to high input impedance and voltagecontrolled gate. So the efforts are continuing to realize a SiC-MOSFET with acceptable channel mobility and oxide reliability. Improving the interface between oxide and $\mathrm{SiC}$ is essential for increasing effective channel mobility and hence reducing device resistance. Double-implanted $4 \mathrm{H}$ $\mathrm{SiC}$ MOSFET reports a channel mobility of $20 \mathrm{~cm}^{2} / \mathrm{V}$.s for $1 \mathrm{kV}$ device resulting in specific on-resistance as low as $6.95 \mathrm{~m} \Omega \mathrm{cm}^{2}$ [9].

For high breakdown voltages, bipolar devices offer low forward voltage drop. For voltages higher than $3 \mathrm{kV}$, SiC PiN diodes offer, in addition to the low forward voltage drop, a faster switching speed making them a good candidate to replace silicon PiN diodes in this voltage class. Unlike Si bipolar junction transistors, SiC BJT can be made with a thinner base region increasing the device current gain $(\beta)$. A power BJT with $\beta$ as high as 134 was demonstrated [10]. Recently, $1200 \mathrm{~V}$ breakdown voltage BJTs are commercially available from TranSiC [11].

Figure 1 shows the latest achievements of various $\mathrm{SiC}$ devices. It is evident that the significant reduction of devices specific on-state resistance will be reflected as an increase on converter efficiency.

\section{SiC Properties for Renewable Energy Applications}

The superior properties of $\mathrm{SiC}$ semiconductor have made it a material of choice for the new generation of power devices. Power devices with switching and conduction characteristics that are closer to ideal switch are being realised using SiC. In addition, the development of such devices impacts many aspects of converter performance and design. Main properties that influence converter design especially for renewable energy sources can be summarised as following.

(i) Low on-resistance: the lower resistance of the devices results in a lower conduction loss and thus a higher converter efficiency.

(ii) Low switching loss: the fact that $\mathrm{SiC}$ switches are capable of switching faster keeping a low switching energy loss implies an improvement of converter efficiency. Moreover, it enables higher switching frequency operation. This leads to smaller and lighter passive elements, that is, inductor and capacitors, increasing the power density of the conversion unit.

(iii) High blocking voltage capability: this is particularly promising for renewables with high power rates such as wind energy. Excising solutions are mostly based on medium DC link voltage $(\sim 700 \mathrm{~V})$ making use of step-up transformer unavoidable for gird connection purpose. Transformerless direct drivewind generator can reduce nacelle weight as well as increase system efficiency and reliability [12]. With megawatt wind turbine, generators with high output voltage $(<20 \mathrm{kV})$ could be employed. SiCbased power conditioning unit (AC-DC-AC) can be design for higher DC link voltages with the help of SiC-GTO or SiC-IGBT technologies. The high blocking voltage of these devices allows for simple and yet more reliable converter design. For example, three-phase inverter using a single high voltage device replaces multi-level converter or the need for 
connecting several devices in series will simplify the converter design and control. This is also beneficial for applications where high DC-link voltage is needed like those found in utility and traction applications.

(iv) High operating temperature: theoretically, SiC devices can operate at junction temperatures up to $700^{\circ} \mathrm{C}$ before its pn junction becomes nonfunctional. However, existing packaging technology prevents such high operating temperature. The highest reported temperature for full $\mathrm{SiC}$ converter built utilizing JFETs and SBDs is $400^{\circ} \mathrm{C}$ [13]. It is important to note here that the high temperature capability has another advantage that it decreases the system heat management demands. For example, this can lead to the use of a smaller heat sink size or facilitate the use of a high temperature coolant cycle, which already exists in the system, like combining converter and engine cooling cycles in a hybrid vehicle system.

\section{SiC JFET for Renewable Applications}

4.1. Device Characterization. The device characterized in the following is an enhancement-mode normally-off vertical JFET [14]. The device has a blocking voltage capability of $1200 \mathrm{~V}$ with maximum datasheet on-resistance of only $63 \mathrm{~m} \Omega$. The JFET structure is free from oxide interfaces that have reliability issues and cause low channel resistivity. Figure 2 shows a cross-sectional view of an enhancementmode vertical SiC JFET. The device exhibits a normallyoff characteristic. This is achieved by $\mathrm{p}$-type gate region implantation (in n-type channel device) that is totally depleted at zero gate voltage, full fabrication details are given in [15]. Having a voltage controlled gate, SiC JFET is potential replacement of Si MOSFET and IGBT. Parasitic elements of this structure are also shown in Figure 2. Gatesource region forms a pn diode. Turning this diode on by applying a positive gate voltage, that consequently leads the depletion region to shrink, is the main requirement for turning on the JFET. Moreover, the parasitic capacitances (Cgs, Cgd, and Cds) affect switching speed. The SiC JFET has no internal body diode so external diode is needed for most applications.

Resistance of drain-source region, usually referred to as on-resistance, is an important performance measure. Thanks to $\mathrm{SiC}$ high critical field, this resistance can be dramatically reduced by achieving high blocking voltage with relatively higher doped and thinner drift region, compared to Si.

Measured on-resistance of the JFET at different temperatures is shown in Figure 3. This increase in device resistance is due to mobility degradation at higher temperatures. In addition, device on-resistance changes with gate voltage. In the case of Figure 3, resistance is measured at gate voltage of $2.65 \mathrm{~V}$.

4.2. Dynamic Characterization. SiC JFET gate has relatively a low threshold voltage, that is, $(\sim 1 \mathrm{~V})$, which makes gate less immune to noise, especially induced voltages across the gate connections. In addition, device gate-source

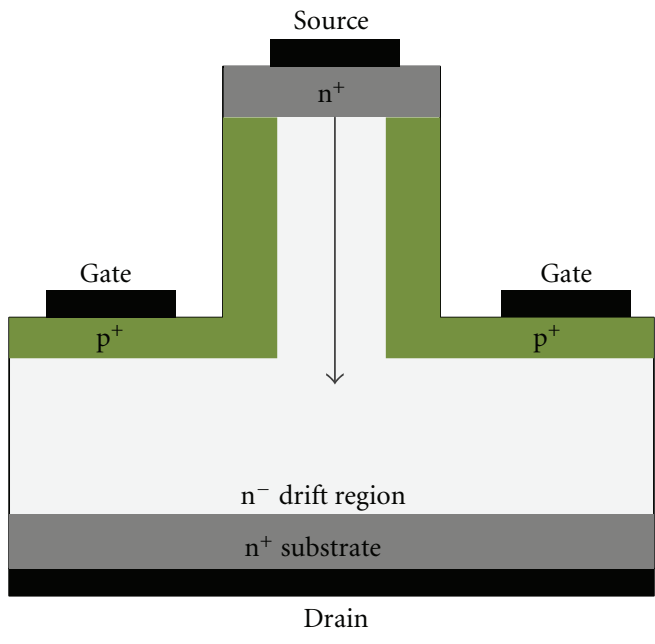

Figure 2: Cross-sectional view of SiC vertical JFET.

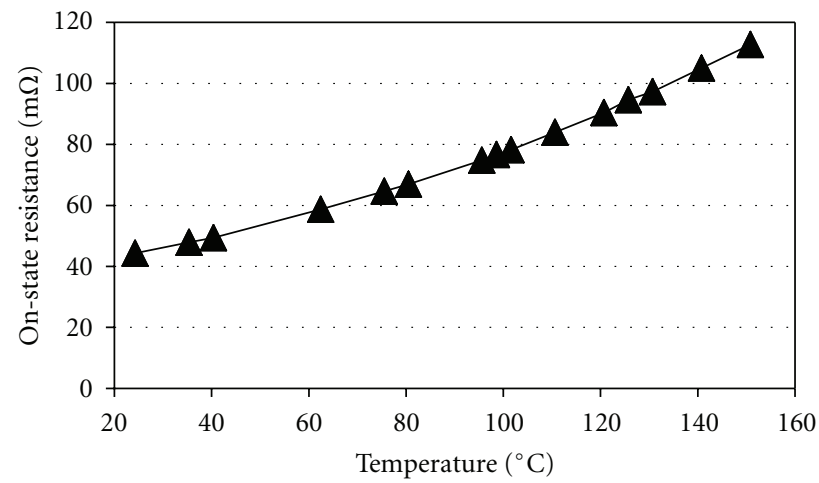

FIGURE 3: On-resistance of SiC JFET versus temperature.

region structure, which is basically a pn-junction, requires more attention when designing the driver. The basic driver requirement is the ability to keep device gate-source diode (Dgs) forwardly biased during the intended on period. Although JFET switching speed in solely determined by its parasitic capacitors, driver ability to charge/discharge these capacitors during on/off transition has a significant influence on switching speed. Improving the dynamic of the driver is done by employing the concept of two-stage driver. The twostage driver fulfills the requirements of driving SiC JFET by quickly providing a charging current for input capacitances $(\mathrm{Cgd}+\mathrm{Cgs})$. This is done by a short gate voltage pulse design to ensure device entering on-state period and lowering onstate resistance without passing excessive current through Dgs. Such current can destroy gate region. The first stage is followed be a lower voltage stage (typically $\sim 3 \mathrm{~V}$ ). This second stage lasts during intended on period. It keeps Dgs forwardly biased and thus the device. In the same time this keeps the gate current at very low level resulting in lower driver losses. The driver circuit is also ensuring a fast turn-off by discharging device capacitances. With negative voltage level during the off-period, gate immunity to noise 


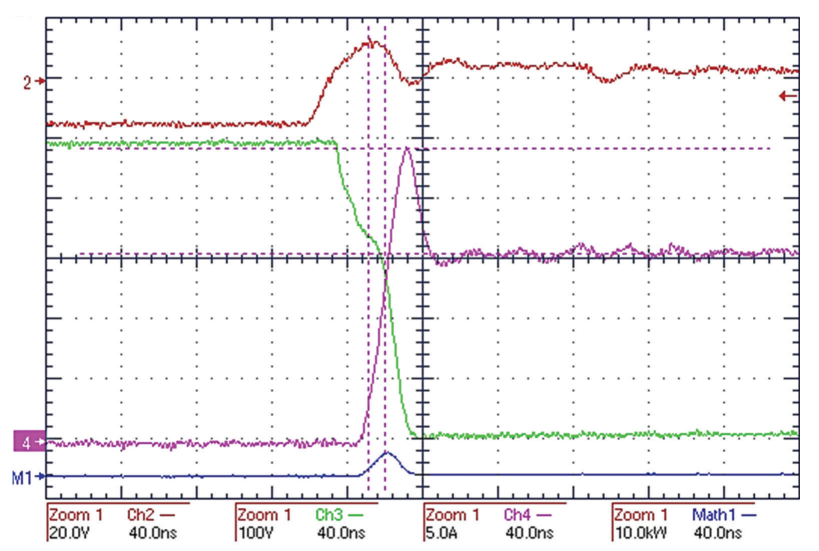

FIgure 4: Turn-on switching of SiC JFET, where $V_{g}($ red), $V_{\mathrm{ds}}$ (green), $I_{\mathrm{d}}$ (maginta), and power(blue).

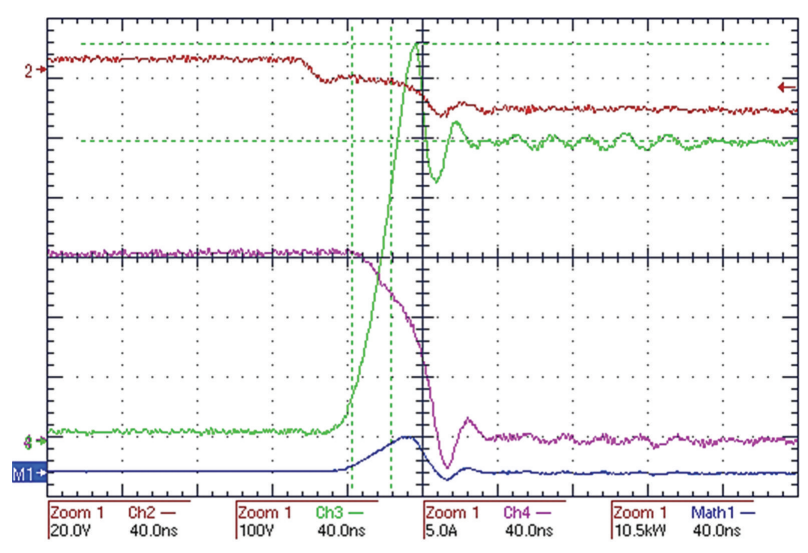

Figure 5: Turn-off switching of SiC JFET, where $V \mathrm{~g}($ red), $V_{\mathrm{ds}}$ (green), $I_{\mathrm{d}}$ (maginta), and power (blue).

can be improved and unwanted turn-on is avoidable. In addition, adding an external capacitance across gate-source will help increasing this immunity. Designing gate driver is challenging due to low threshold voltage and induced gate voltages as a result of overshoots in voltage and current waveforms at high switching speed. It is therefore important to use twisted conductor to connect driver circuit with device gate.

Figures 4 and 5 show turn-on and turn-off oscillograms. Switching waveforms are recorded during a standard double pulse test with clamped inductive load performed at drainsource voltage $V_{\mathrm{ds}}=500 \mathrm{~V}$ and drain current $I_{\mathrm{d}}=15 \mathrm{~A}$ with gate resistance $R g=3.5 \Omega$. A SiC Schottky diode was used as a clamping diode. The test facilitates characterising the dynamic characteristics of the device where switching times and energies can be measured. This test emulates hard switching conditions such as grid connected inverters and motor drives.

The recorded turn-on and turn-off times are $48 \mathrm{nsec}$ and $56 \mathrm{nsec}$, respectively. These values demonstrate device ability for fast switching. However, the fast switching speed makes the converter operation very sensitive to circuit layout. Optimized layout is necessary to avoid any EMI problems

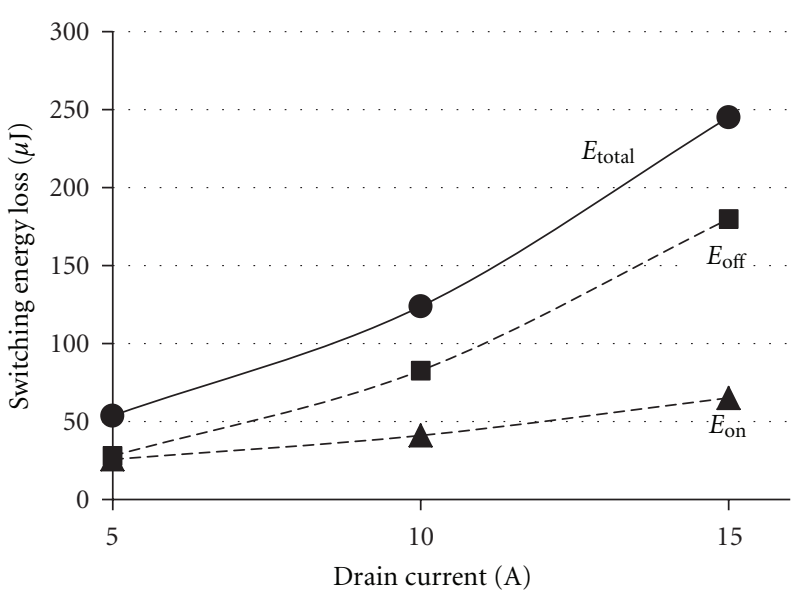

FIGURE 6: SiC JFET switching energy components versus drain current at $25^{\circ} \mathrm{C}$.

related to this fast switching. For example, stray inductance should be minimized to avoid unwanted oscillation at high $d i / d t$. The test setup used here utilizes multilayer PCB with strip conductor keeping distances between DC-link capacitor and device terminals minimal. Stray inductance appears at the JFET drain acts as a turn-on snubber causing a longer turn-on transition.

4.3. Switching Loss. Due to fast switching speed and the unipolar operation, SiC JFET is expected to have low switching losses. Figure 6 shows the device turn-on energy $\left(E_{\text {on }}\right)$, turn-off energy $\left(E_{\text {off }}\right)$, and total switching energy versus drain current. Switching energies are calculated by integrating power loss waveform during turn-on and turnoff periods. The test is performed with $V_{\mathrm{ds}}=500 \mathrm{~V}$ and an ambient temperature of $25^{\circ} \mathrm{C}$. The recorded $E_{\text {on }}$ and $E_{\text {off }}$ at drain current of $15 \mathrm{~A}$ were $62 \mu \mathrm{J}$ and $181 \mu \mathrm{J}$, respectively. Total energy loss witnesses a slight increase as junction temperature increases. An increase of only 3\% of total energy loss was recorded at temperature of $150^{\circ} \mathrm{C}$.

To theoretically evaluate potential inverter efficiency, device power loss is estimated based on datasheet and measurement values. Equations used for this loss estimation are given in [16]. Total power dissipation of a three-phase inverter configuration uses SiC JFETs and Schottky diodes is shown in Figure 7. This calculation assumes an inverter with a DC-link voltage of $500 \mathrm{~V}$ and output peak current of $15 \mathrm{~A}$. During this calculation of power dissipation, switching, conduction and driver circuit losses were included. SiC JFET demonstrates the ability to operate at higher switching frequency with acceptable efficiency. The total power loss of the inverter is compared when the SiC JFET is replaced with the state-of-the-art Si IGBT (IKW15T120) from Infineon [17]. The device has the same voltage rating of $1200 \mathrm{~V}$ as well as current rating of $15 \mathrm{~A}$. By comparing the total power losses of the two inverters, the losses are higher in case of $\mathrm{Si}$ IGBT is used. Moreover, power losses increases prohibitively with the switching frequency. So, employing the SiC JFET in inverter design will improve efficiency and enables high 


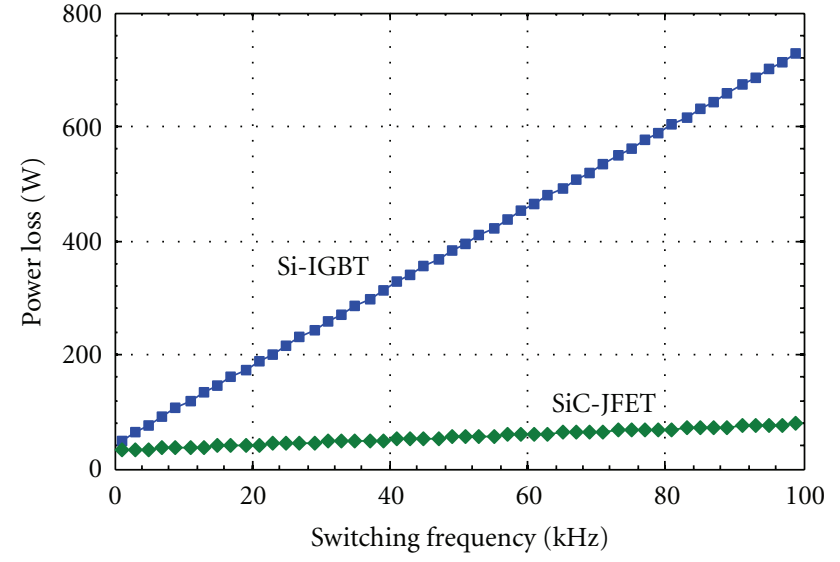

FIGURE 7: Calculated power loss in three-phase inverter for SiC JFET versus Si IGBT as a function of switching frequency using the same freewheeling diode at $\left(25^{\circ} \mathrm{C}\right)$.

switching frequency operation. Solar inverters can highly benefit from these characteristics which are expected to bring energy cost down by reducing the weight and the size of both passive elements (inductance and capacitance) and the heat sink. This in return will increase market acceptance of this energy resource. Other high performance conversion systems such as hybrid vehicle, electric vehicle, and auxiliary power supply will also benefit from the demonstrated features of $\mathrm{SiC}$ devices.

\section{Conclusions}

In this paper a review of $\mathrm{SiC}$ devices status and impact on renewable energy applications is given. The needs of renewable energy conversion systems, such as inverter used in solar and wind applications, were matched to the superior features offered by SiC devices. The focus is on one of the most successful SiC devices, namely, JFET. The device combines low conduction and switching losses with fast switching capability. This makes it a candidate for high efficiency conversion systems. By increasing the switching frequency, the weight, size and cost of passive elements can be reduced. Moreover, device ability to operate at higher temperatures enables designs with reduced heat sink size and thus further increase in power density. These features are expected to reduce the cost of renewable energy systems which eventually will lead to more market acceptance of renewable energy sources.

\section{Acknowledgments}

The authors thank Subhadra Tiwari and Øyvind H. Snefjellå for providing help during the laboratory measurements.

\section{References}

[1] B. Burger and D. Kranzer, "Extreme high efficiency PV-power converters," in Proceedings of the 13th European Conference on Power Electronics and Applications, EPE '09, pp. 1-13, September 2009.

[2] U. Singisetti, W. M. Hoi, J. S. Speck, and U. K. Mishra, "Enhancement-mode N-Polar GaN MOS-HFET With 5-nm GaN channel, $510-\mathrm{mS} / \mathrm{mm} \mathrm{g}_{\mathrm{m}}$, and $0.66-\Omega \cdot \mathrm{mm} \mathrm{R}_{\mathrm{on}}$," IEEE Electron Device Letters, vol. 33, no. 1, pp. 26-28, 2012.

[3] C. Y. Tsai, T. L. Wu, and A. Chin, "High-performance GaN MOSFET With High- $\kappa \mathrm{LaAlO}_{3} / \mathrm{SiO}_{2}$ gate dielectric," IEEE Electron Device Letters, vol. 33, no. 1, pp. 35-37, 2012.

[4] T. Kimoto, T. Urushidani, S. Kobayashi, and H. Matsunami, "High-voltage (>1KV) SiC schottky barrier diodes with low on-resistances," IEEE Electron Device Letters, vol. 14, no. 12, pp. 548-550, 1993.

[5] D. T. Morisette, J. A. Cooper Jr., M. R. Melloch et al., "Static and dynamic characterization of large-area high-currentdensity SiC Schottky diodes," IEEE Transactions on Electron Devices, vol. 48, no. 2, pp. 349-352, 2001.

[6] R. Singh, J. A. Cooper Jr., M. R. Melloch, T. P. Chow, and J. W. Palmour, "SiC power Schottky and PiN diodes," IEEE Transactions on Electron Devices, vol. 49, no. 4, pp. 665-672, 2002.

[7] K. Rueschenschmidt, M. Treu, R. Rupp et al., "SiC JFET: currently the best solution for an unipolar SiC high power switch," Materials Science Forum, vol. 600-603, pp. 901-906, 2009.

[8] J. B. Casady, D. C. Sheridan, R. L. Kelley, V. Bondarenko, and A. Ritenour, "A comparison of $1200 \mathrm{~V}$ normally-OFF \& normally-ON vertical trench SiC power JFET devices," Materials Science Forum, vol. 679-680, pp. 641-644, 2011.

[9] A. Saha and J. A. Cooper, "A 1-kV 4H-SiC power DMOSFET optimized for low ON-resistance," IEEE Transactions on Electron Devices, vol. 54, no. 10, pp. 2786-2791, 2007.

[10] K. Nonaka, A. Horiuchi, Y. Negoro et al., "A new high current gain $4 \mathrm{H}-\mathrm{SiC}$ bipolar junction transistor with suppressed surface recombination structure: SSR-BJT," Materials Science Forum, vol. 615-617, pp. 821-824, 2009.

[11] TranSiC, 2011, http://www.transic.com/.

[12] M. A. Parker, C. H. Ng, L. Ran, P. Tavner, and E. Spooner, "Power control of direct drive wind turbine with simplified conversion stage \& transformerless grid interface," in Proceedings of the 41st International Universities Power Engineering Conference (UPEC '06), pp. 65-68, September 2006.

[13] T. Funaki, J. C. Balda, J. Junghans et al., "Power conversion with SiC devices at extremely high ambient temperatures," IEEE Transactions on Power Electronics, vol. 22, no. 4, pp. 13211329, 2007.

[14] SJEP120R100 Datasheet, http://www.semisouth.com.

[15] A. Ritenour, D. C. Sheridan, V. Bondarenko, and J. B. Casady, "Performance of $15 \mathrm{~mm}^{2} 1200 \mathrm{~V}$ normally-off SiC VJFETs with 120 A saturation current," Materials Science Forum, vol. 645-648, pp. 937-940, 2010.

[16] M. H. Bierhoff and F. W. Fuchs, "Semiconductor losses in voltage source and current source IGBT converters based on analytical derivation," in Proceedings of the 35th IEEE Annual Power Electronics Specialists Conference (PESC '04), pp. 28362842, June 2004.

[17] IKW15T120 Datashee, http://www.Infineon.com/. 

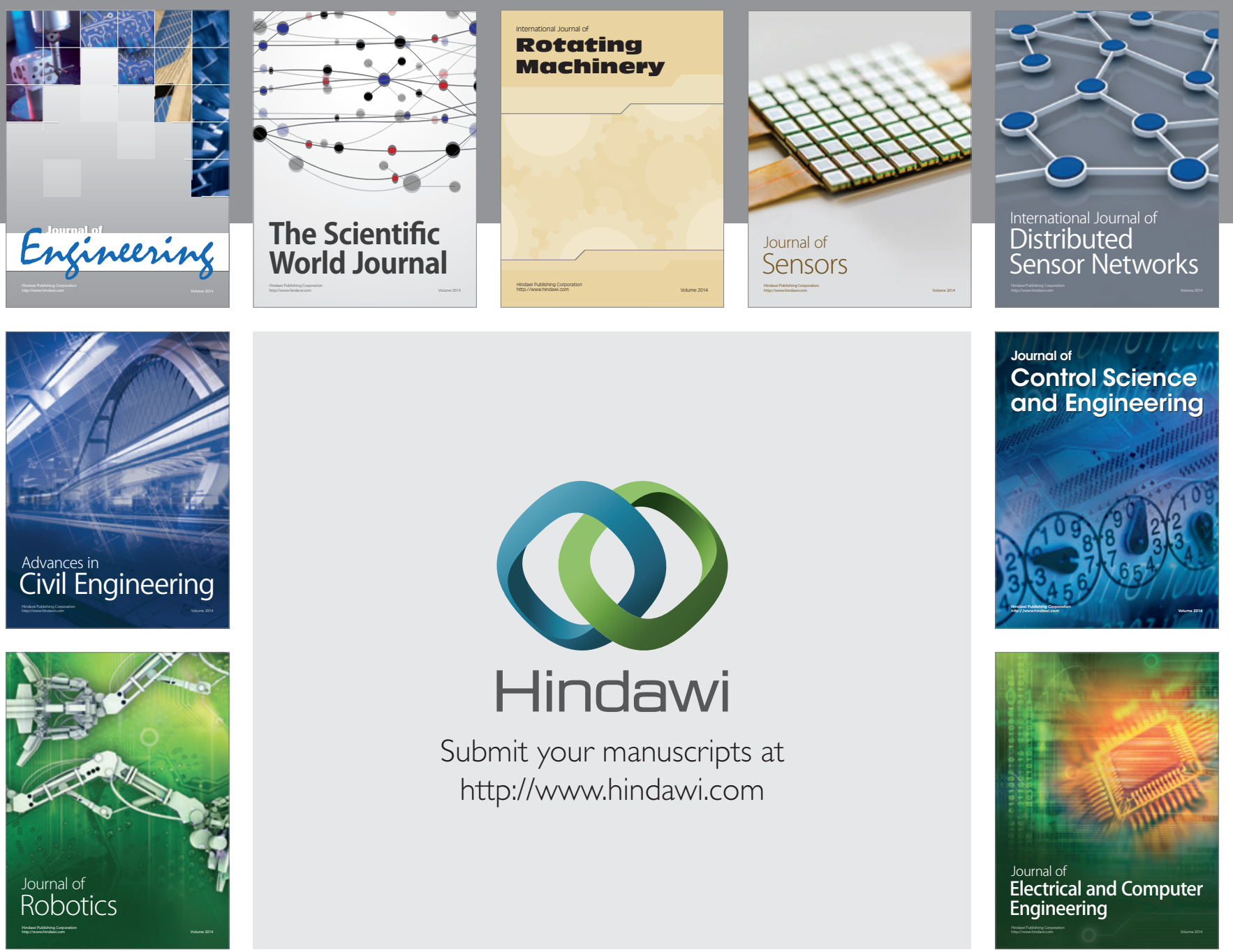

Submit your manuscripts at

http://www.hindawi.com
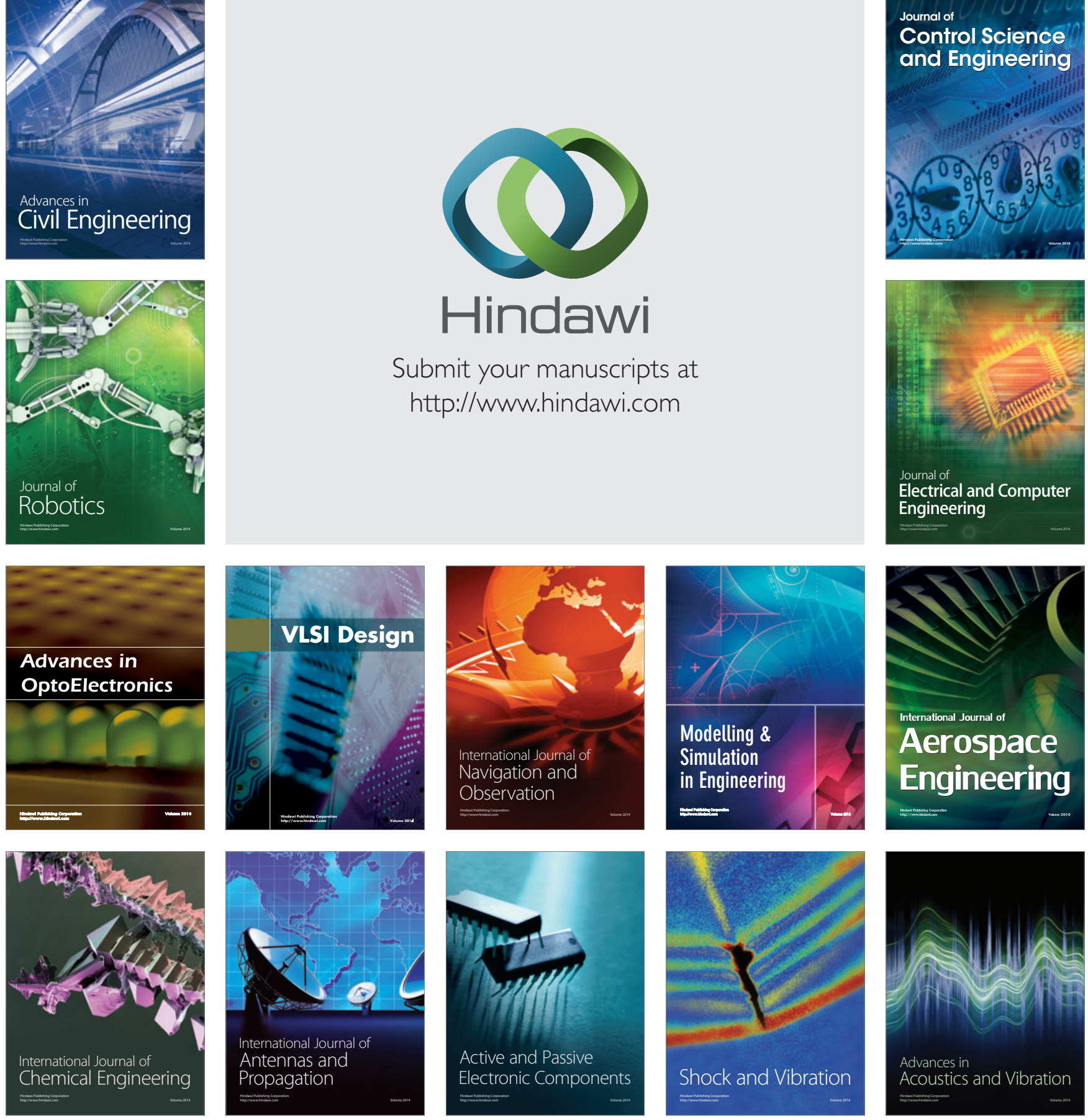